\title{
Arsenic Trioxide Inhibits Cell Growth and Invasion via Down- Regulation of Skp2 in Pancreatic Cancer Cells
}

\author{
Jian-Kun Gao ${ }^{1,2}$, Li-Xia Wang², Bo Long ${ }^{3}$, Xian-Tao Ye², Jing-Na Su², Xu-Yuan \\ Yin $^{2}$, Xiu-Xia Zhou ${ }^{2 *}$, Zhi-Wei Wang ${ }^{2 *}$
}

\begin{abstract}
Arsenic trioxide (ATO) has been found to exert anti-cancer activity in various human malignancies. However, the molecular mechanisms by which ATO inhibits tumorigenesis are not fully elucidated. In the current study, we explored the molecular basis of ATO-mediated tumor growth inhibition in pancreatic cancer cells. We used multiple approaches such as MTT assay, wound healing assay, Transwell invasion assay, annexin V-FITC, cell cycle analysis, RT-PCR and Western blotting to achieve our goal. We found that ATO treatment effectively caused cell growth inhibition, suppressed clonogenic potential and induced G2-M cell cycle arrest and apoptosis in pancreatic cancer cells. Moreover, we observed a significant down-regulation of Skp2 after treatment with ATO. Furthermore, we revealed that ATO regulated Skp2 downstream genes such as FOXO1 and p53. These findings demonstrate that inhibition of Skp2 could be a novel strategy for the treatment of pancreatic cancer by ATO.
\end{abstract}

Keywords: Arsenic trioxide - Skp2 - cell growth - apoptosis - pancreatic cancer

Asian Pac J Cancer Prev, 16 (9), 3805-3810

\section{Introduction}

Pancreatic cancer (PC) is one of the most prevalent malignancies worldwide (Torre et al., 2015). Although multiple treatment strategies such as surgery, chemotherapeutics and radiation have been used in clinic, PC keeps the fourth deadliest disease in the United States (Siegel et al., 2015). The majority of PC patients become refractory to standard chemotherapeutic drugs such as gemcitabine and 5-FU (5-fluorouracil) or their combination (Michl and Gress, 2013). Notably, the molecular mechanism of PC development is largely unclear. Therefore, it is pivotal to explore the new targets and to develop efficient pharmacological agents for the treatment of PC.

Emerging evidence has demonstrated that the F-box protein Skp2 (S-phase kinase associated protein 2) plays a critical role in the development and progression of human cancers including PC (Wang et al., 2014b). It has been well documented that Skp2 exerts its oncogenic activity through targeting and degrading its ubiquitination targets including p21 (Yu et al., 1998), p27 (Tsvetkov et al., 1999), p57 (Kamura et al., 2003), E-cadherin (Inuzuka et al., 2012), and FOXO1 (Huang et al., 2005a). In line with this note, Skp2 plays a key role in cell growth, apoptosis, cell cycle progression, migration, invasion, metastasis, and angiogenesis (Wang et al., 2012a). Moreover, high expression of Skp2 was observed in PC (Einama et al., 2006), breast tumors (Radke et al., 2005; Fujita et al., 2008), nasopharyngeal carcinoma (Wang et al., 2014a), and prostate cancer (Wang et al., 2012b). Strikingly, highlevel Skp2 was correlated with the extent of lymph node metastasis, higher histological grade, and poorer patient outcome in PC patients (Einama et al., 2006). Furthermore, Skp2 conferred resistance of PC cells towards the TRAIL (tumor necrosis factor-related apoptosis-inducing ligand) treatment (Schuler et al., 2011). Recently, it has been revealed that inactivation of Skp2 inhibited prostate tumorigenesis through mediating JARID1B (Jumonji AT rich interactive domain $1 \mathrm{~B})$ ubiquitination ( $\mathrm{Lu}$ et al., 2015). These reports indicated that Skp2 could be a promising therapeutic molecular target in PC.

Arsenic trioxide (ATO) has been found to exert its anti-cancer activity in a variety of human cancers (Zhou, 2012). ATO has been used as a Chinese traditional medicine for more than 2400 years (Wang and Chen, 2008). More importantly, ATO has been introduced as an anticancer agent in patients with acute promyelocytic leukemia (APL) since 1970s (Mi et al., 2012). In 2000, the FDA approved ATO injection, TrisenoxTM, as a first-line chemotherapeutic agent for the treatment of APL (de The and Chen, 2010). Interestingly, ATO has been observed to have promising activities in various solid tumors, such as liver cancer, pancreatic cancer, breast cancer, and gastric

\footnotetext{
${ }^{I}$ Department of Basic Medical Sciences, Sichuan College of Traditional Chinese Medicine, ${ }^{3}$ Infectious Diseases Department, Mianyang 404 Hospital, Mianyang, Sichuan, ${ }^{2}$ Cyrus Tang Hematology Center and Collaborative Innovation Center of Hematology, Jiangsu Institute of Hematology, The First Affiliated Hospital, Soochow University, Suzhou, China*For correspondence: zwang6@bidmc. harvard.edu,xxzhou@suda.edu.cn
} 
cancer (Subbarayan and Ardalan, 2014). Although some studies have determined the mechanisms of ATO-mediate tumor growth inhibition, the exact molecular mechanisms are not fully elucidated.

Herein, we investigated the anti-cancer potential of ATO against PC cell lines. We found that ATO treatment led to cell growth inhibition, suppressed clonogenic potential, induced apoptosis and G2-M cell cycle arrest. Consistently, ATO reduced cell migration and invasion in PC cell lines. Mechanistically, we observed a significant down-regulation of Skp2 in PC cells treated with ATO. More importantly, we unraveled that ATO regulated Skp2 downstream genes such as FOXO1 and p53. These findings demonstrate that down-regulation of Skp2 by ATO could be an imperative approach for the treatment of PC.

\section{Materials and Methods}

\section{Cell culture and experiment reagents}

Human PC cell lines Patu8988 and Panc-1 were obtained from ATCC and maintained in standard cell culture conditions at $37^{\circ} \mathrm{C}$ in a humidified $5 \% \mathrm{CO}_{2}$ atmosphere. Antibodies against Skp2, FOXO1, P53, $\beta$-actin and the secondary antibodies were obtained from Santa Cruz Biotechnology (Santa Cruz, CA, USA). ATO and all other chemicals were bought from Sigma (St. Louis, MO). ATO was dissolved in $1 \mathrm{mM} \mathrm{NaOH}$ to make $1 \mathrm{mM}$ stock solution and was added directly to the media at different concentrations.

\section{Cell viability studies by MTT assay}

The Patu8988 and Panc-1 cells $\left(4 \times 10^{3}\right)$ were seeded in a 96-well culture plate for overnight. Then, cells were treated with various concentrations of ATO for $48 \mathrm{~h}$ and $72 \mathrm{~h}$. At the end of treatment period, $10 \mu \mathrm{L}$ of Reagent MTT ( $5 \mathrm{mg} / \mathrm{mL}$ in PBS) was added to each well. After 2 $\mathrm{h}$ incubation, $100 \mu \mathrm{L}$ of DMSO was added to each well and further incubated for 10 minutes in dark. The color intensity was measured by SpectraMax M5 microplate fluorometer (Molecular Device, US) at $490 \mathrm{~nm}$.

\section{Clonogenic assay}

To test the survival of cells treated with ATO, Panc-1 and Patu8988 cells were plated $\left(3 \times 10^{5}\right.$ per well) in a sixwell plate and incubated overnight. After $72 \mathrm{~h}$ exposure to various concentrations of ATO, the viable cells were counted and plated in $100 \mathrm{~mm}$ dishes in a range of 3,000 cells per plate. The cells were then incubated for 21 days at $37^{\circ} \mathrm{C}$ in a humidified $5 \% \mathrm{CO}_{2}$ atmosphere. All the colonies were stained with $2 \%$ crystal violet.

\section{Wound healing assay}

Wound healing assay was conducted to examine the capacity of cell migration. Panc- 1 and Patu 8988 cells were seeded in a six-well plate at the concentration of $2 \times 10^{6}$ cells per well. The wound was generated in the cells with 90-95\% confluent by scratching the surface of the plates with a sterile pipette tip. The cells were then incubated in the absence or presence of ATO for 20h, and then photographed with an Olympus microscope.

\section{Transwell invasion assay}

The invasive capacity of Panc- 1 and Patu8988 cells was performed using Transwell Filter $(8 \mu \mathrm{m}$ pore size, Corning) with Matrigel (BD Biosciences). Briefly, around $1.5 \times 10^{5}$ Panc- 1 and $5 \times 10^{4}$ Patu8988 cells in serum-free media were transferred in each upper chamber in the presence or absence of ATO. 0.5 milliliter of culture medium with $10 \%$ FBS was added into each bottom chamber in the presence or absence of ATO. After incubation for $20 \mathrm{~h}$, the cells in the upper chamber were removed (the upper surfaces of the Transwell chambers were scraped with cotton swabs), and the invaded cells were fixed and stained using the Wright's-Giemsa stain set (Jiancheng Scientific, Nanjing, China). The stained cells were photographed and counted under a light microscope in six randomly-selected fields.

\section{Flow cytometry and cell cycle analysis}

Cells were seeded in 6-well plate and incubated overnight. Then, the cells were exposed to ATO and grown for $72 \mathrm{~h}$. At the end of treatment period, cells were collected and fixed with ice-cold $70 \%(\mathrm{v} / \mathrm{v})$ ethanol for $24 \mathrm{~h}$. After centrifugation at $3000 \times \mathrm{g}$ for $5 \mathrm{~min}$, the cell pellets were washed with PBS (pH 7.4) and resuspended in PBS containing propidium iodide $(50 \mu \mathrm{g} / \mathrm{mL})$ at $37^{\circ} \mathrm{C}$ for $30 \mathrm{~min}$. Next, DNase-free RNase A $(100 \mu \mathrm{g} / \mathrm{mL})$ was added. Samples were then incubated at room temperature for $30 \mathrm{~min}$, and DNA contents were determined by flow cytometry using a flow cytometer (BD, San Jose, CA).

\section{Annexin V-FITC method for apoptosis analysis}

Annexin V-FITC apoptosis detection kit (Biouniqure, China) was used to measure the apoptotic cells. Panc-1 and Patu 8988 cells were incubated in the presence or absence of ATO for $48 \mathrm{~h}$. Cell were collected by centrifugation and then were resuspend in $500 \mu \mathrm{l}$ of binding buffer. After that, $5 \mu 1$ of Annexin V-FITC and $5 \mu 1$ of PI (propidium iodide) were added. All the samples were kept in the dark for $15 \mathrm{~min}$ at room temperature. Finally, the stained cells were analyzed using a flow cytometer (BD, San Jose, CA).

\section{Protein extraction and western blotting}

For protein extraction, ATO treated cells were harvested and lysed with cell lysis buffer (Cell Signaling, Danvers, MA). The protein concentrations were measured using the BCA protein assay kit (CWBIO, Beijing, China). Proteins were fractionated using sodium dodecyl sulfatepolyacrylamide gel electrophoresis (SDS-PAGE), and the gels were transferred onto nitrocellulose membrane. The membranes were blocked with $4 \%$ nonfat dried milk or bovine serum albumin in $1 \times \mathrm{PBS}$ containing $0.1 \%$ Tween-20, and then incubated over night at $4^{\circ} \mathrm{C}$ with appropriate primary antibodies. The membranes were washed 3 times with PBS-T, and subsequently incubated with the secondary antibodies for $1 \mathrm{~h}$ at room temperature. The protein bands were detected using the enhanced chemiluminesence detection system.

\section{Data analysis}

Data are represented as mean \pm SD for the absolute values or percentage of controls as indicated in the 
Arsenic Trioxide Inhibits Cell Growth and Invasion via Down-Regulation of Skp2 in Pancreatic Cancer Cells

vertical axis legend of figures. The statistic significance of differential findings between experimental groups and control groups was statistically evaluated using GraphPad StatMate software (GraphPad Software, Inc., San Diego, CA). $p$ value $<0.05$ was considered statistically significant.

\section{Results}

Effects of ATO on the viability of PC cells by MTT assay It has been reported that ATO inhibited cell growth in HPAF, MiaPaCa-2 and Panc-1 cells (Li et al., 2002). In order to further investigate the influence of ATO on PC cell growth, we evaluated the cell viability of Patu 8988 and Panc- 1 cells treated with ATO for $48 \mathrm{~h}$ or $72 \mathrm{~h}$ by MTT assay. In line with previous report (Li et al., 2002), we found that ATO inhibited cell growth in a dosedependent manner in both Panc-1 and Patu8988 PC cell lines (Figure 1A and 1B). Specifically, the treatment of Panc- 1 cells for $2-3$ days with $1 \mu \mathrm{M}, 2 \mu \mathrm{M}$, and $3 \mu \mathrm{M}$ led to cell growth inhibition (Figure 1A). However, Patu8988 cells were more resistant to ATO because up to $3 \mu \mathrm{M}$ of ATO treatment resulted in cell growth inhibition (Figure 1B). Moreover, we observed that $2 \mu \mathrm{M}$ and $6 \mu \mathrm{M}$ of ATO caused about $50 \%$ cell growth inhibition in Panc-1 and Patu 8988 cells, respectively. Therefore, we selected $1 \mu \mathrm{M}$, $2 \mu \mathrm{M}$ ATO for Panc- 1 cells and $3 \mu \mathrm{M}, 6 \mu \mathrm{M}$ for Patu 8988 cells in the following studies.

\section{Effects of ATO on cell survival by clonogenic assay}

To further confirm the effects of ATO on cell growth, cells were treated with ATO and assessed for cell viability by clonogenic assay. ATO treatment resulted in a significant inhibition of colony formation of Panc-1 and Patu8988 cells when compared with control (Figure 1C and 1D). Moreover, we observed a dose-dependent inhibition of colony formation by ATO. Overall, the results from clonogenic assay was consistent with the MTT data (Figure 1A and 1B), suggesting that ATO inhibited cell growth in Panc-1 and Patu8988 cells.

\section{Inhibition of cell migration by ATO in PC cells}

In order to examine whether ATO could prevent migratory potential in Panc- 1 and Patu8988 PC cells, we conducted wound healing assays in cells treated with ATO. The wound healing assay demonstrated that ATO reduced cell motility in both Panc-1 and Patu8988 cells (Figure $2 \mathrm{~A}$, and $2 \mathrm{~B}$ ). Moreover, ATO inhibited cell migration in a dose-dependent manner in both PC cells. Next, we investigated whether ATO could inhibit cell invasion in PC cells.

\section{Inhibition of cell invasion by ATO in PC cells}

To further confirm the function of ATO in cell motility, we measured the cell invasion capacities in PC cells with ATO treatment using Matrigel invasion chamber assay. We found that ATO significantly retarded the cell invasion in both Panc-1 and Patu8988 cells (Figure 3A). The value of the invaded Panc-1 cells was decreased about 2-fold by $2 \mu \mathrm{M}$ ATO treatment compared with that of control (Figure 3B). In addition, the invasion of Patu8988 cells was inhibited about 2.5-fold and 4-fold by $3 \mu \mathrm{M}$ and $6 \mu \mathrm{M}$ ATO
A

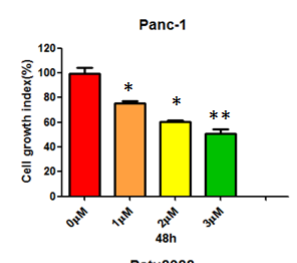

B

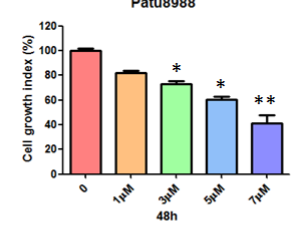

C

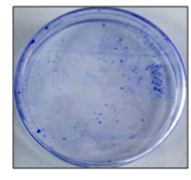

Control

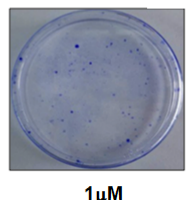

$1 \mu \mathrm{M}$
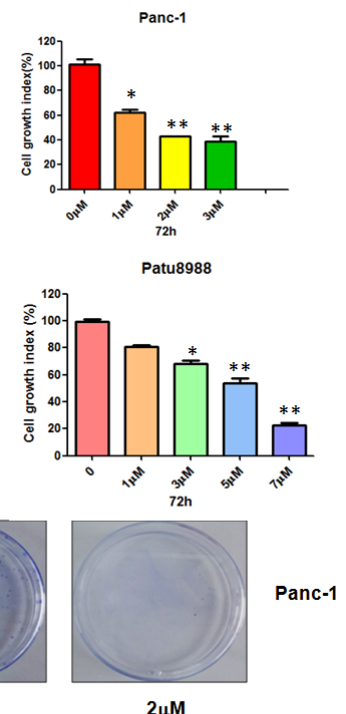

D
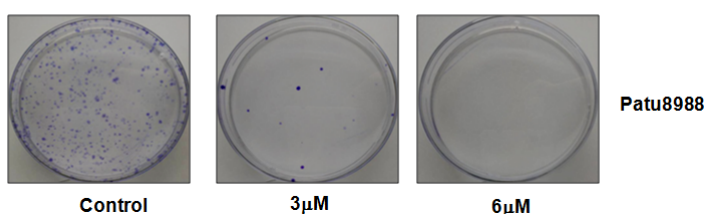

Figure 1. Effect of ATO on PC Cell Growth. A-B. Cells were seeded in 96-well plates at a density of 4,000 per well and treated with indicated concentrations of ATO for 48 and $72 \mathrm{~h}$. After treatment, cell viability was determined using the MTT assay as described in Materials and Methods. Vertical bars indicate the mean of three independent experiments; bars, SD. ${ }^{*} p<0.05,{ }^{*} p<0.01$, compared with the control. C-D. Cell treated with varied concentrations of ATO for $72 \mathrm{~h}$ were evaluated by the clonogenic assay. Photographic differences in colony formation in Panc-1 and Patu8988 untreated and treated with ATO are shown

A

on

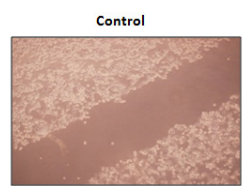

${ }_{1 \mu \mathrm{M}}$

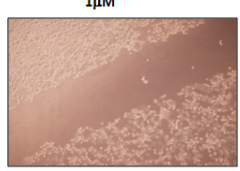

$2 \mu \mathrm{M}$

$20 \mathrm{~h}$
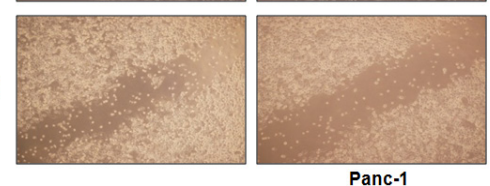

Control

B
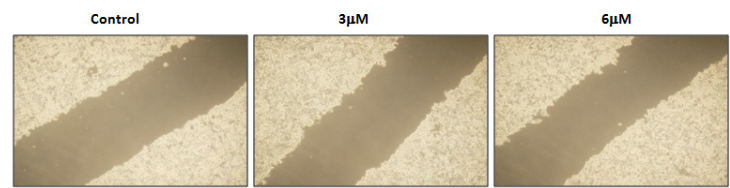

$20 \mathrm{~h}$
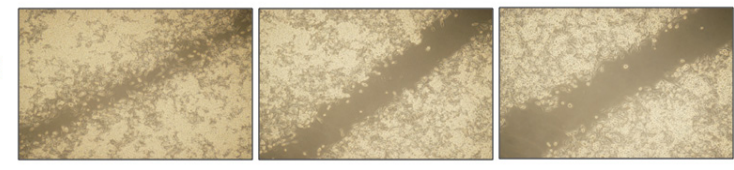

Patu8988

Figure 2. ATO Inhibits Cell Migration in PC Cells. Inhibition of cell migration by ATO was performed using the wound healing assay in Panc-1 cells (A) and Patu8988 cells (B). The wound was generated in the cells with $90-95 \%$ confluent by scratching the surface of the plates with a sterile pipette tip. The cells were then incubated in the absence and presence of ATO for $20 \mathrm{~h}$ and wound healing images were captured using an Olympus microscope 

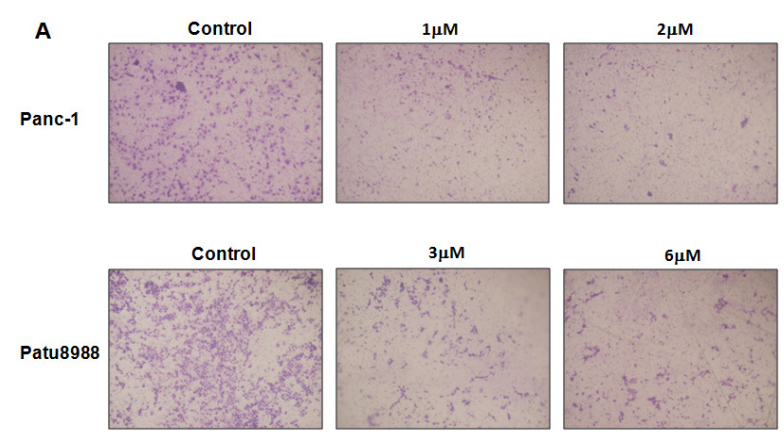

B
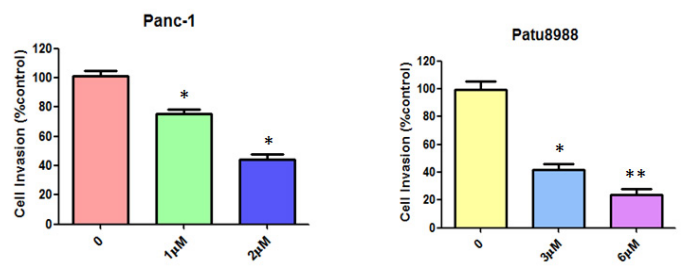

Figure 3. ATO Inhibits Cell Invasion in PC Cells. A. Cells that invaded to the lower surface of the insert over a period of $20 \mathrm{~h}$ were stained with Giemsa solution. The stained cells were photographed under a light microscope. B. The stained cells were counted under a light microscope in six randomly-selected fields. Quantitative measurement of invaded cells was assessed. The number of cells was normalized against the number of cells in the corresponding control cells. The error bars represent mean $\pm \mathrm{SD}$ $(\mathrm{n}=6) . * p<0.01, * * p<0.05$ vs control
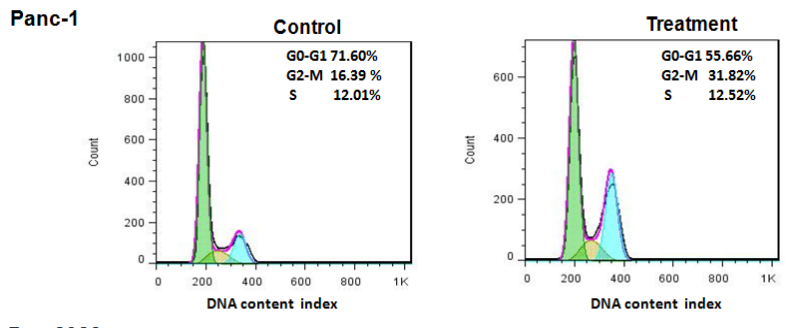

Patu8988
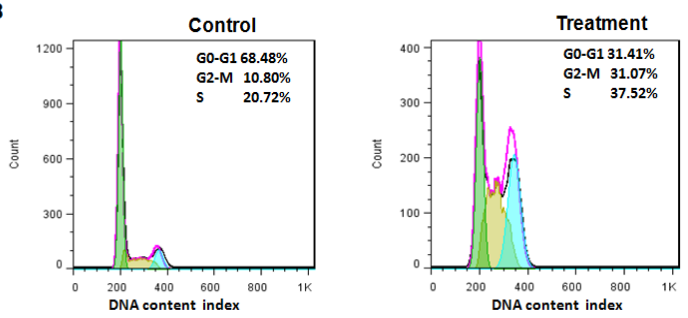

Figure 4. ATO Induces Cell Cycle Arrest at G2-M

Phase in PC Cells. Panc-1 and Patu8988 cells were exposed to indicated concentrations of ATO for $72 \mathrm{~h}$ and were harvested for cell cycle analysis using propidium iodide staining. $\mathrm{X}$ axis, DNA content; $\mathrm{Y}$ axis, the number of nuclei

treatment, respectively (Figure 3B). This finding indicated that ATO retarded the invasive potential of PC cells.

\section{ATO induces cell cycle arrest in PC cells}

In order to verify whether ATO abrogated cell cycle progression, propidium iodide flow cytometry assay was performed. Panc-1 and Patu 8988 cells were exposed to ATO for $72 \mathrm{~h}$ and analyzed for cell cycle distribution by flow cytometry (FACS) analysis after propidium iodide staining of the cellular DNA. We found that ATO induced an accumulation of cells in the G2-M phase fractions in comparison with untreated control cells (Figure 4). The G2-M phase fraction increased from $16.39 \%$ in control cells to $31.82 \%$ and from $10.80 \%$ in control cells to
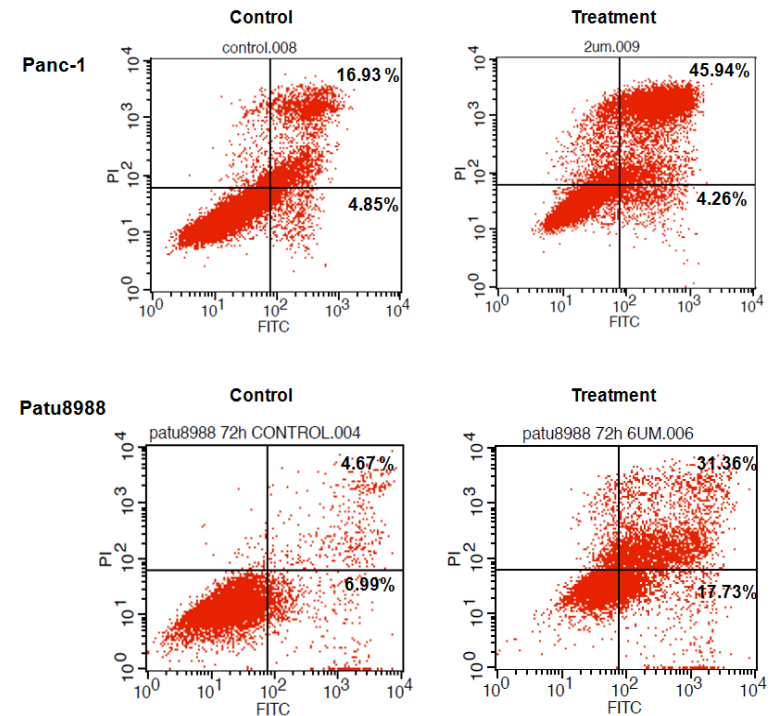

Figure 5. ATO Induces Apoptosis in PC Cells. Panc-1 and Patu8988 cells were exposed to different concentrations of ATO for $72 \mathrm{~h}$. Apoptosis were determined by Annexin V-FITC
A
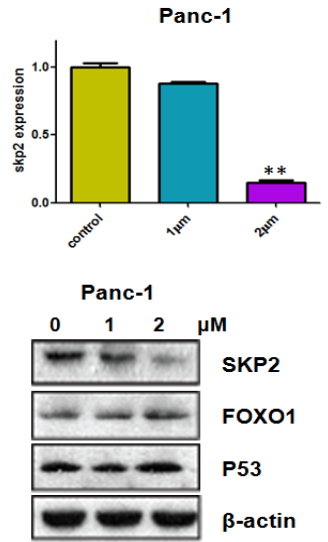

C
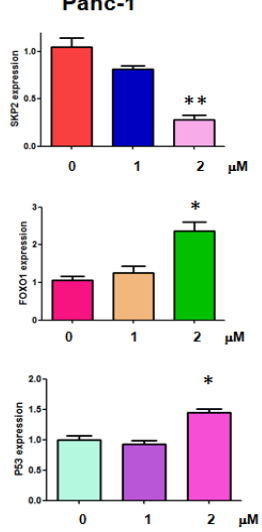

Patu8988

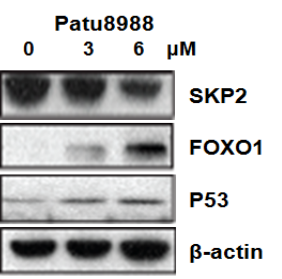

Patu8988
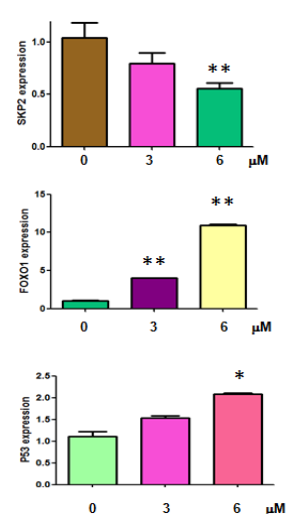

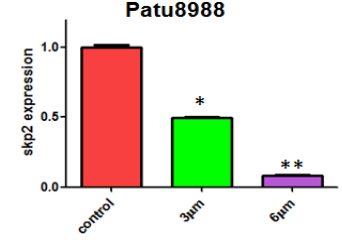

Figure 6. Effect of ATO on the Expression of Skp2. A. Real-time RT-PCR was performed to measure the Skp2 mRNA levels in PC cells treated with ATO for 72h. B. The protein levels of Skp2 and its downstream genes were detected by Western blotting in PC cells treated with ATO for $72 \mathrm{~h}$. C. Quantitative results are illustrated for panel B. $* p<0.05, * * p<0.01 v s$ control

$31.07 \%$ in ATO-treated Panc-1 cells and Patu8988 cells, respectively (Figure 4). These findings further solidify the case for ATO as an effect agent against PC.

\section{ATO induces apoptosis in PC cells}

To determine whether cell growth inhibition by ATO in 
PC cells is due to induction of apoptosis, the cell apoptosis assay using Annexin V/PI staining was performed. By staining cells with annexin V-FITC and PI, we found that the percentage of apoptotic cells increased from $16.93 \%$ in the control to $45.94 \%$ and from $4.67 \%$ in control cells to $31.36 \%$ in ATO-treated Panc-1 and Patu8988 cells (Figure 5). Our results showed that ATO treatment resulted in a statistically significant increase in the percentage of apoptotic cells in both PC cell lines, suggesting that ATO could be a potent apoptosis inducer in PC cells.

\section{Down-regulation of Skp2 by ATO in PC cells}

It has been well documented that Skp2 was critically involved in the development and progression of PC (Einama et al., 2006). To further understand the molecular mechanism underlying the anti-tumor activities of ATO in PC cells, alterations in the cell survival pathway with special emphasis in Skp2 were investigated using Realtime RT-PCR and Western blotting analysis, respectively. Our Real-time RT-PCR results showed that ATO remarkably inhibited the Skp2 mRNA levels (Figure 6A). Notably, we observed that ATO significantly suppressed the expression of Skp2 at protein levels compared with control (Figure 6B, 6C). It has been reported that tumor suppressor FOXO1 is a substrate of Skp2 (Huang et al., 2005b). Additionally, Skp2 has been found to attenuate p53 function in human cancers (Chander et al., 2010). In line with these findings, our results revealed that ATO treatment enhanced accumulation of tumor suppressor FOXO1 and p53 in PC cells (Figure 6B and 6C).

\section{Discussion}

A number of evidence has demonstrated that ATO inhibited cell proliferation and induced apoptosis in PC cells (Li et al., 2002; Li et al., 2003). For example, one study has shown that ATO treatment inhibited cell growth and caused apoptosis through activation of caspase- 3 and PARP (poly ADP ribose polymerase) cleavage in three human PC cell lines, HPAF, MiaPaCa- 2 and PANC-1 (Li et al., 2002). Moreover, this group found that ATO triggered apoptosis through down-regulation of Bcl-2 and Mcl-1, as well as upregulation of Bax (Li et al., 2003). Furthermore, ATO markedly increased the expression of GADD45 and GADD153 in PC cells (Li et al., 2004). It has also been reported that ATO inhibited proliferation and induced apoptosis in multiple myeloma cells (Elmahi et al., 2013). Recently, Han et al. found that ATO inhibited viability of PC stem cells in vitro and in vivo through binding to SHH (sonic hedgehog)-Gli (Han et al., 2013). Consistent with these findings, we observed that ATO suppressed cell growth and induced apoptosis in Panc-1 and Patu8988 cells. Additionally, our results demonstrated that ATO inhibited cell migration and invasion in PC cells. These findings suggest that ATO may be valuable for the treatment of PC.

Recent studies have highlighted the critical role of Skp2 in pancreatic tumorigenesis (Schuler et al., 2011). Thus, targeting Skp2 could have considerable therapeutic benefits for the treatment of PC patients with aberrant activation of Skp2 (Chan et al., 2014). To this end, several small inhibitors of Skp2 have been developed and showed anti-tumor activity (Wu et al., 2012). For instance, the compound CpdA prevented p27 from recruitment to Skp2 ligase complex, leading to the accumulation of p27 and subsequent tumor inhibition (Chen et al., 2008). Another Skp2 inhibitor, SMIP0004, was observed to downregulate Skp2 and to stabilize p27 (Rico-Bautista et al., 2010). More recently, Compound 25 (also known as SZL-P1-41) has been developed through a high-throughput screening as a Skp2 inhibitor (Chan et al., 2013). Compound 25 selectively suppressed Skp2 E3 ligase activity and exhibited potent antitumor activities in vitro and in vivo (Chan et al., 2013). Interestingly, mounting evidence has demonstrated that several natural agents including curcumin, lycopene, silibinin, epigallocatechin-3-gallate, quercetin, and Vitamin D3 could inhibit the expression of Skp2 in human cancers (Yang and Burnstein, 2003; Roy et al., 2007; Huang et al., 2008; Huang et al., 2011). Recently, one study indicated that resveratrol suppressed Skp2mediated ubiquitylation and 26S proteasome-dependent degradation of p27 in oesophageal adenocarcinoma (Fan et al., 2014). Our current study revealed that ATO inhibited the expression of Skp2 in PC cells, indicating that ATO could be a potential Skp2 inhibitor. However, further mouse modeling studies are required to validate the antitumor activity by ATO as a Skp2 inhibitor.

It is noteworthy that ATO has no activity in PC patients who develop progressive disease after gemcitabine (Kindler et al., 2008). This suggests that ATO could need to combine with other agents to have better treatment benefit (Subbarayan and Ardalan, 2014). In other words, ATO could be a chemosensitizer in combination therapy in patients diagnosed with PC. In support of this notion, it has been reported that combination therapy with ATO and parthenolide has an augmented anti-tumor effect on PC through induction of reactive oxygen species generation and apoptosis (Wang et al., 2009). Consistently, combination treatment of ATO and gemcitabine retarded tumor growth in a xenograft model in PC (Han et al., 2013). Therefore, it is necessary to investigate whether a combination of ATO and other chemotherapeutic drugs have better benefits in PC.

In summary, we have provided experimental evidence that supports the role of ATO as a potential anti-tumor agent for PC. Mechanistically, we propose that ATO attenuated Skp2 expression and subsequent upregulation of its downstream genes such as p53 and FOXO1, leading to inhibition of cell proliferation, invasion and induction of apoptosis in PC cells. Based on these findings, we believe that down-regulation of Skp2 by ATO could be a promising therapeutic molecular target in PC.

\section{Acknowledgements}

This work is supported by grant from National Natural Science Foundation of China (NSFC 81172087) and a project funded by the priority academic program development of Jiangsu higher education institutions. This work is also supported in part by Sichuan Province Education Department and Sichuan College of Traditional Chinese Medicine (No. 13ZA0333). 


\section{References}

Chan CH, Morrow JK, Li CF, et al (2013). Pharmacological inactivation of Skp2 SCF ubiquitin ligase restricts cancer stem cell traits and cancer progression. Cell, 154, 556-68.

Chan CH, Morrow JK, Zhang S, et al (2014). Skp2: a dream target in the coming age of cancer therapy. Cell cycle, 13, 679-80.

Chander H, Halpern M, Resnick-Silverman L, et al (2010). Skp2B attenuates p53 function by inhibiting prohibitin. EMBO Reports, 11, 220-5.

Chen Q, Xie W, Kuhn DJ, et al (2008). Targeting the p27 E3 ligase SCF (Skp2) results in p27- and Skp2-mediated cellcycle arrest and activation of autophagy. Blood, 111,4690-9.

de The H, Chen Z (2010). Acute promyelocytic leukaemia: novel insights into the mechanisms of cure. Nature Reviews. Cancer, 10, 775-83.

Einama T, Kagata Y, Tsuda H, et al (2006). High-level Skp2 expression in pancreatic ductal adenocarcinoma: correlation with the extent of lymph node metastasis, higher histological grade, and poorer patient outcome. Pancreas, 32, 376-81.

Elmahi AY, Niu C, Li W, et al (2013). Effects of arsenic trioxide alone and in combination with bortezomib in multiple myeloma RPMI 8266 cells. Asian Pac J Cancer Prev, 14, 6469-73.

Fan GH, Wang ZM, Yang X, et al (2014). Resveratrol inhibits oesophageal adenocarcinoma cell proliferation via AMPactivated protein kinase signaling. Asian Pac J Cancer Prev, 15, 677-82.

Fujita T, Liu W, Doihara H, et al (2008). Dissection of the APCCdh1-Skp2 cascade in breast cancer. Clin Cancer Res, 14, 1966-75.

Han JB, Sang F, Chang JJ, et al (2013). Arsenic trioxide inhibits viability of pancreatic cancer stem cells in culture and in a xenograft model via binding to SHH-Gli. OncoTargets Therapy, 6, 1129-38.

Huang H, Regan KM, Wang F, et al (2005a). Skp2 inhibits FOXO1 in tumor suppression through ubiquitin-mediated degradation. P Natl Acad Sci USA, 102, 1649-54.

Huang H, Regan KM, Wang F, et al (2005b). Skp2 inhibits FOXO1 in tumor suppression through ubiquitin-mediated degradation. Proc Natl Acad Sci USA, 102, 1649-54.

Huang HC, Lin CL, Lin JK (2011). 1,2,3,4,6-penta-O-galloylbeta-D-glucose, quercetin, curcumin and lycopene induce cell-cycle arrest in MDA-MB-231 and BT474 cells through downregulation of Skp2 protein. J Agric Food Chem, 59, 6765-75.

Huang HC, Way TD, Lin CL, et al (2008). EGCG stabilizes p27kip1 in E2-stimulated MCF-7 cells through downregulation of the Skp2 protein. Endocrinology, 149,5972-83.

Inuzuka H, Gao D, Finley LW, et al (2012). Acetylationdependent regulation of Skp2 function. Cell, 150, 179-93.

Kamura T, Hara T, Kotoshiba S, et al (2003). Degradation of p57Kip2 mediated by SCFSkp2-dependent ubiquitylation. Proc Natl Acad Sci USA, 100, 10231-6.

Kindler HL, Aklilu M, Nattam S, et al (2008). Arsenic trioxide in patients with adenocarcinoma of the pancreas refractory to gemcitabine: a phase II trial of the University of Chicago Phase II Consortium. Am J Clin Oncol, 31, 553-6.

Li X, Ding X, Adrian TE (2002). Arsenic trioxide inhibits proliferation and induces apoptosis in pancreatic cancer cells. Anticancer Res, 22, 2205-13.

Li X, Ding X, Adrian TE (2003). Arsenic trioxide induces apoptosis in pancreatic cancer cells via changes in cell cycle, caspase activation, and GADD expression. Pancreas, 27, 174-9.

Li X, Ding X, Adrian TE (2004). Arsenic trioxide causes redistribution of cell cycle, caspase activation, and GADD expression in human colonic, breast, and pancreatic cancer cells. Cancer investigation, 22, 389-400.

Lu W, Liu S, Li B, et al (2015). SKP2 inactivation suppresses prostate tumorigenesis by mediating JARID1B ubiquitination. Oncotarget, 6, 771-88.

Mi JQ, Li JM, Shen ZX, et al (2012). How to manage acute promyelocytic leukemia. Leukemia, 26, 1743-51.

Michl P, Gress TM (2013). Current concepts and novel targets in advanced pancreatic cancer. Gut, 62, 317-26.

Radke S, Pirkmaier A, Germain D (2005). Differential expression of the F-box proteins Skp2 and Skp2B in breast cancer. Oncogene, 24, 3448-58.

Rico-Bautista E, Yang CC, Lu L, et al (2010). Chemical genetics approach to restoring p27Kip1 reveals novel compounds with antiproliferative activity in prostate cancer cells. $B M C$ Biol 8, 153.

Roy S, Kaur M, Agarwal C, et al (2007). p21 and p27 induction by silibinin is essential for its cell cycle arrest effect in prostate carcinoma cells. Mol Cancer Ther, 6, 2696-707.

Schuler S, Diersch S, Hamacher R, et al (2011). SKP2 confers resistance of pancreatic cancer cells towards TRAIL-induced apoptosis. Int J Oncol, 38, 219-25.

Siegel RL, Miller KD, Jemal A (2015). Cancer statistics, 2015. Ca Cancer J Clin, 65, 5-29.

Subbarayan PR, Ardalan B (2014). In the war against solid tumors arsenic trioxide need partners. J Gastrointest Cancer, 45, 363-71.

Torre LA, Bray F, Siegel RL, et al (2015). Global cancer statistics, 2012. Ca Cancer J Clin, 65, 87-108.

Tsvetkov LM, Yeh KH, Lee SJ, et al (1999). p27 (Kip1) ubiquitination and degradation is regulated by the SCF (Skp2) complex through phosphorylated Thr187 in p27. Current Biol, 9, 661-4.

Wang G, Chan CH, Gao Y, et al (2012a). Novel roles of Skp2 E3 ligase in cellular senescence, cancer progression, and metastasis. Chinese J Cancer, 31, 169-77.

Wang J, Huang Y, Guan Z, et al (2014a). E3-ligase Skp2 predicts poor prognosis and maintains cancer stem cell pool in nasopharyngeal carcinoma. Oncotarget, 5, 5591-601.

Wang W, Adachi M, Zhang R, et al (2009). A novel combination therapy with arsenic trioxide and parthenolide against pancreatic cancer cells. Pancreas, 38, 114-23.

Wang Z, Gao D, Fukushima H, et al (2012b). Skp2: a novel potential therapeutic target for prostate cancer. Biochim Biophys Acta, 1825, 11-7.

Wang Z, Liu P, Inuzuka H, et al (2014b). Roles of F-box proteins in cancer. Nature Reviews Cancer, 14, 233-47.

Wang ZY, Chen Z (2008). Acute promyelocytic leukemia: from highly fatal to highly curable. Blood, 111, 2505-15.

Wu L, Grigoryan AV, Li Y, et al (2012). Specific small molecule inhibitors of Skp2-mediated p27 degradation. Chem Biol, 19, 1515-24.

Yang ES, Burnstein KL (2003). Vitamin D inhibits G1 to S progression in $\mathrm{LNCaP}$ prostate cancer cells through $\mathrm{p} 27 \mathrm{Kip} 1$ stabilization and Cdk2 mislocalization to the cytoplasm. $J$ Biol Chem, 278, 46862-8.

Yu ZK, Gervais JL, Zhang H (1998). Human CUL-1 associates with the SKP1/SKP2 complex and regulates p21 (CIP1/ WAF1) and cyclin D proteins. Proc Natl Acad Sci USA, 95, 11324-9.

Zhou J (2012). Arsenic trioxide: an ancient drug revived. Chinese Med J, 125, 3556-60. 